Pacific Journal of Mathematics

RATIONAL FUNCTIONS WITH POSITIVE COEFFICIENTS 


\title{
RATIONAL FUNCTIONS WITH POSITIVE COEFFICIENTS, POLYNOMIALS AND UNIFORM APPROXIMATIONS
}

\author{
Peter B. Borwein
}

\begin{abstract}
Upper bounds are established for the uniform approximation of continuous functions on $[1,0]$ by rational functions with positive coefficients. These bounds are obtained by rewriting polynomials with no positive roots as rational functions with positive coefficients.
\end{abstract}

1. Introduction. The uniform closure in $C[1,0]$ of the set of polynomials with positive coefficients includes only those functions analytic in the unit disc whose power series expansions have nonnegative coefficients. The uniform closure of the set of rational functions with positive coefficients consists of all continuous functions which are never negative on $[0,1]$. This is a consequence of the following interesting factorization theorem.

THEOREM 1. (E. Meissner [3].) Suppose that $p$ is a polynomial with real coefficients and that $p(x)>0$ for $x>0$. Then there exists a rational function $r(x)$ with nonnegative coefficients so that $p(x)=r(x)$.

We will provide an accurate bound for the degree of the above $r$ in terms of the degree of $p$ and some knowledge of the location of the roots of $p$. We will also derive some estimates concerning how efficiently polynomials can be approximated on $[0,1]$ by rational functions with positive coefficients. We will exploit these results to prove a number of approximation theorems. For instance: if $f$ is analytic in some neighborhood of $[0,1]$ and positive on $[0,1]$, then there exists a sequence of rational functions $\left\{r_{n}\right\}$ where each $r_{n}$ is of degree $n$ and has nonnegative coefficients so that $\left\|f-r_{n}\right\|_{[0,1]}=$ $0\left(\alpha^{-\sqrt{n}}\right)$ for some $\alpha>1$.

We employ the following notation. Let $\Pi_{n}$ denote the polynomials with real coefficients of degree at most $n$. Let $\Pi_{n}^{+}$be the sub class of $\Pi_{n}$ whose elements have nonnegative coefficients. Let $R_{n}^{++}$denote those rational functions $p_{n} / q_{n}$ where $p_{n}, q_{n} \in \Pi_{n}^{+}$. For $f \in C[a, b]$ define

$$
\begin{aligned}
& \Pi_{n}(f:[a, b])=\inf _{p \in \Pi_{n}}\|f-p\|_{[a, b]} \\
& \Pi_{n}^{+}(f:[a, b])=\inf _{p \in \Pi^{+}}\|f-p\|_{[a, b]} \\
& R_{n}^{++}(f:[a, b])=\inf _{r \in R^{++}}\|f-r\|_{[a, b]}
\end{aligned}
$$


where \|\|$_{[a, b]}$ is the supremum norm on $[a, b]$. We note that all the above infimums are attained.

2. Expressing polynomials as rational functions with nonnegative coefficients. The first two results of this section are concerned with expressing quadratic polynomials as rational functions in $R_{m}^{++}$where $m$ is as small as possible. The final theorem is an extension of these results to general polynomials.

TheOREM 2. Suppose that $\alpha, \beta>0$ and suppose that $x^{2}-\alpha x+\beta$ has no positive roots. Then

(a) for each $\varepsilon>0$ there exists a constant $A_{\varepsilon}$ so that

$$
x^{2}-\alpha x+\beta=r_{m}(x)
$$

where

$$
r_{m} \in R_{m}^{++} \text {and } m \leqq A_{\varepsilon}\left[\frac{1}{4-\alpha^{2} / \beta}\right]^{1 / 2+\varepsilon} \text {. }
$$

(b) for $\varepsilon=1 / 14$,

$$
x^{2}-\alpha x+\beta=r_{m}(x)
$$

where

$$
r_{m} \in R_{m}^{++} \text {and } m \leqq 20\left[\frac{1}{4-\alpha^{2} / \beta}\right]^{1 / 2+1 / 14}
$$

Proof. The quadratic $x^{2}-\alpha x+\beta$ has no positive root if and only if $\alpha^{2}<4 \beta$. We set $c=\alpha^{2} / \beta$ and note that $0<c<4$. Consider

$$
\begin{aligned}
\left(x^{2}-\alpha x+\beta\right)\left(x^{2}+\alpha x+\beta\right) & =x^{4}+\left(2 \beta-\alpha^{2}\right) x^{2}+\beta^{2} \\
& =x^{4}+\beta(2-c) x^{2}+\beta^{2} .
\end{aligned}
$$

If $c \leqq 2$ we have the desired factorization. In general we proceed as follows:

Define $C_{n}$ inductively by

$$
C_{0}=c^{1 / 2} \text { and } C_{n+1}=2-C_{n}^{2} .
$$

Let

$$
p_{n}(x)=x^{2^{n+1}}+\beta^{2^{n-1}} C_{n} x^{2^{n}}+\beta^{2^{n}}
$$

and let

$$
\overline{p_{n}}(x)=x^{2^{n+1}}-\beta^{2^{n-1}} C_{n} x^{2^{n}}+\beta^{2^{n}} .
$$

Note that, by (2) 


$$
\begin{aligned}
p_{n}(x) \overline{p_{n}}(x) & =x^{2^{n+2}}-{\beta^{2 n}}^{2} C_{n}^{2} x^{2^{n+1}}+2{\beta^{2 n}}^{2^{2^{n-1}}}+\beta^{2^{n+1}} \\
& =x^{2^{n+2}}+\beta^{2^{n}} C_{n+1} x^{2^{n+1}}+\beta^{2^{n+1}} \\
& =p_{n+1}(x)
\end{aligned}
$$

Consider the smallest $n$ (if it exists) so that $C_{n}$ is nonnegative. Then, by (1) and (3)

$$
\left(x^{2}-\alpha x+\beta\right)\left(x^{2}+\alpha x+\beta\right)=p_{1}
$$

and

$$
p_{1} \cdot \overline{p_{1}} \cdot \overline{p_{2}} \cdots \overline{p_{n-1}}=p_{n}
$$

where $\overline{p_{1}} \cdots \cdot \overline{p_{n-1}} \in \Pi_{\left(2^{n+1}-4\right)}^{+}$since each $C_{k}<0$ for $k<n$ and where $p_{n} \in \Pi_{2^{n+1}}^{+}$since $C_{n} \geqq 0$. Thus, we have

$$
x^{2}-\alpha x+\beta=\frac{p_{n}}{\left(x^{2}+\alpha x+\beta\right) \overline{p_{1}} \cdot \overline{p_{2}} \cdots \cdot \overline{p_{n-1}}} \in R_{2^{n+1}}^{++} .
$$

Since $0<c^{1 / 2}<2$ we deduce that $C_{n} \rightarrow 1$. We wish to find a small $n$ as a function of $C$, so that

$$
C_{n} \geqq 0
$$

Suppose that

$$
C_{1}, \cdots, C_{n}<0
$$

Then

$$
C_{n}=2-\left(C_{n-1}\right)^{2}<0
$$

implies

$$
\left(C_{n-1}\right)^{2}>2 \text { and }-C_{n-1}>2^{1 / 2}
$$

implies

$$
\left(C_{n-2}\right)^{2}-2>2^{1 / 2} \text { and }-C_{n-2}>\left(2+2^{1 / 2}\right)^{1 / 2}
$$

and by iteration

$$
\left.c>2+\left(2+\cdots\left(2+2^{1 / 2}\right)^{1 / 2}\right)^{1 / 2} \cdots\right)^{1 / 2}=\delta_{n}
$$

where (equivalently) $\delta_{1}=2$ and $\delta_{n}=2+\delta_{n-1}^{1 / 2}$.

We are reduced to finding an $n$ so that $\delta_{n}>c=\alpha^{2} / \beta$ since, for such an $n(6)$ is contradicted and hence, (5) is satisfied.

Consider 
(8)

$$
\begin{aligned}
4-\delta_{n} & =2-\delta_{n-1}^{1 / 2}=\frac{4-\delta_{n-1}}{2+\delta_{n-1}^{1 / 2}}=\frac{4-\delta_{n-2}}{\left(2+\delta_{n-1}^{1 / 2}\right)\left(2+\delta_{n-2}^{1 / 2}\right)} \\
& \vdots \\
& =\frac{2}{\left(2+\delta_{n-1}^{1 / 2}\right)\left(2+\delta_{n-2}^{1 / 2}\right) \cdots\left(2+\delta_{1}^{1 / 2}\right)} \leqq \frac{2}{\left(2+2^{1 / 2}\right)^{n-1}} \leqq \frac{7}{\left(2+2^{1 / 2}\right)^{n}}
\end{aligned}
$$

It is now sufficient to pick $n$ so that

$$
\frac{7}{\left(2+2^{1 / 2}\right)^{n}} \leqq 4-\frac{\alpha^{2}}{\beta} \text {. }
$$

A suitable choice is

$$
n=1+\text { int. part }\left[\frac{\log _{2}\left[\frac{7}{4-\frac{\alpha^{2}}{\beta}}\right]}{\log _{2}\left(2+2^{1 / 2}\right)}\right] \leqq 1+\frac{4}{7} \log _{2}\left[\frac{7}{4-\frac{\alpha^{2}}{\beta}}\right] .
$$

We deduce from (4) that

$$
x^{2}-\alpha x+\beta \in R_{2^{n+1}}^{++}
$$

where

$$
2^{n+1} \leqq 4\left[\frac{7}{4-\frac{\alpha^{2}}{\beta}}\right]^{4 / 7} \leqq 20\left[\frac{1}{4-\frac{\alpha^{2}}{\beta}}\right]^{1 / 2+1 / 14}
$$

This completes (b). Part (a) is proved analogously with the observation that in (8), for $k<n$,

$$
\left|4-\delta_{n}\right| \leqq \frac{7}{\left(2+\delta_{k}^{1 / 2}\right)^{n-k}} .
$$

Since $\hat{o}_{n} \rightarrow 4$, we can replace (9) by

$$
\frac{F_{\varepsilon}}{(4-\varepsilon)^{n}} \leqq 4-\frac{\alpha^{2}}{\beta}
$$

and the result follows as above.

The bound in Theorem 2 is "essentially" correct.

THEOREM 3. Let $\alpha_{k}=2$ and $\beta_{k}=1+1 / k^{2}$. If

$$
x^{2}-\alpha_{k} x+\beta_{k}=r_{m} \in R_{m}^{++}
$$




$$
m \geqq \sqrt{2}\left[\frac{1}{4-\frac{\alpha_{k}^{2}}{\beta_{k}}}\right]
$$

Proof. We first show that if $p_{n} \in \Pi_{n}^{+}$then $p_{n}$ has no roots in $T_{n}=\{z:|\arg (z)|<\pi / n\} . \quad$ Suppose $p_{n}(z)=\sum_{n=0}^{n} a_{n} z^{n}$ where $a_{n} \geqq 0$. Let $\zeta \in\{0<\arg (z)<\pi / n\}$. Then $a_{h}(\zeta)^{h} \in\{0<\arg (z)<h \pi / n\}$ and hence, $p_{n}(\zeta) \in\{\operatorname{im}(z)>0\}$. Thus, $p_{n}$ has no roots in $T_{n}$.

The quadratic $x^{2}-\alpha_{k} x+\beta_{k}$ has a root at $1+i / k \in T_{k}$ and we deduce that if $x^{2}-\alpha_{k} x+\beta_{k}=r_{m} \in R_{m}^{++}$then $m>k$. We finish the result by observing that

$$
\sqrt{2}\left[\frac{1}{4-\frac{\alpha_{k}^{2}}{\beta_{k}}}\right]^{1 / 2}=\frac{k\left(1+1 / k^{2}\right)^{1 / 2}}{\sqrt{2}} \leqq k .
$$

THEOREM 4. Suppose $p_{n} \in \Pi_{n}$ has no roots in the region $\Omega(1 / h)=$ $\{z:|\arg (z)|<1 / h\}$ and suppose that $p_{n}(x)>0$ for $x>0$. Then,

(a) for each $\varepsilon>0$ there exists a constant $B_{\varepsilon}$, depending only on $\varepsilon$, so that

$$
p_{n}=r_{m} \in R_{m}^{++} \text {where } m \leqq B_{\varepsilon} h^{(1+\varepsilon)} n .
$$

(b) for $\varepsilon=1 / 7$,

$$
p_{n}=r_{m} \in R_{m}^{++} \text {where } m \leqq 10 h^{8 / 7} \cdot n \text {. }
$$

Proof. Let $x^{2}-\alpha x+\gamma$ be a quadratic factor of $p_{n}$. We assume $\alpha, \gamma<0$ since otherwise $x^{2}-\alpha x+\gamma$ has either nonnegative coeffcients or a nonnegative root. We proceed to replace, using Theorem 2 , each such factor by an element of $R_{k}^{++}$.

Set $\gamma=1 / 4\left(1 / h^{2}+1\right) \alpha^{2}+\delta$ and set $\beta=1 / 4\left(1 / h^{2}+1\right) \alpha^{2}$. Since $x^{2}-\alpha x+\gamma$ has no roots in $\Omega(1 / h)$ was see that $\left|\alpha^{2}-4 \gamma\right|^{1 / 2} \geqq \alpha / h$ and $4 \gamma \geqq\left(1 / h^{2}+1\right) \alpha^{2}$ from which we deduce that $\delta \geqq 0$. Consider $x^{2}-\alpha x+\beta$. By Theorem 2(b) $x^{2}-\alpha x+\beta-r_{k} \in R_{k}^{++}$where

$$
k \leqq 20\left[\frac{1}{4-\frac{\alpha^{2}}{\beta}}\right]^{1 / 2+1 / 14}=20\left[\frac{h^{2}\left(1 / h^{2}+1\right)}{4}\right]^{4 / 7} \leqq 20 h^{8 / 7}
$$

We now replace $x^{2}-\alpha x+\gamma$ by $r_{k}+\delta$. Since there are a maximum of $n / 2$ such quadratic terms to replace, we have

$$
p_{n}=r_{m} \in R_{m}^{++} \text {where } m \leqq 20 h^{8 / 7}(n / 2)=10 h^{8 / 7} n .
$$

This completes part (b). Part (a) is proved analogously using 
Theorem 2(a) instead of $2(\mathrm{~b})$.

3. Approximating polynomials. We estimate how efficiently polynomials in the class P.P.C. can be approximated by rationals with positive coefficients. A polynomial is in the class P.P.C. (polynomials with positive coefficients in $x$ and $(1-x)$, see [1]) if it can be written $\Sigma a_{k i} x^{k}(1-x)^{i}$ where $a_{k i} \geqq 0$. We use this estimate and Theorem 4 to approximate polynomials with no roots in a region containing $[0,1]$. We adopt the notation R.P.C. (rationals with positive coefficients in $x$ and $(1-x)$ ) for those rational functions which are a quotient of two elements of the class P.P.C.

Lemma 1. Suppose $p_{n}=\sum_{k+i \leq n} a_{k i} x^{k}(1-x)^{i}$ is a P.P.C. of degree n. Then there exists $r(x) \in R_{n m}^{++}$so that for $x \in[0,1)$,

$$
\left|r(x)-p_{n}(x)\right| \leqq \frac{n x^{m} p_{n}(x)}{(1-x)^{n}}
$$

Proof. We observe that for $x \in[0,1)$,

$$
\begin{gathered}
\left|(1-x)-\frac{1}{1+x \cdots+x^{m-1}}\right|=\left|(1-x)-\frac{1-x}{1-x^{m}}\right| \\
=\left|\frac{x^{m}(1-x)}{1-x^{m}}\right| \leqq x^{m} .
\end{gathered}
$$

Since $a^{i}-b^{i}=(a-b)\left(a^{i-1}+a^{i-2} b+\cdots+a b^{i-2}+b^{i-1}\right)$,

$$
\left|(1-x)^{i}-\frac{1}{\left(1+x+\cdots+x^{m-1}\right)^{2}}\right| \leqq i x^{m} .
$$

Let

$$
s_{m}(x)=\frac{1}{1+x+\cdots+x^{m-1}}
$$

and consider

$$
r \cdot(x)=\sum_{k+i \geqq n} a_{k i} x^{k}\left(s_{m}\right)^{i} .
$$

Each term of the above sum can be brought to the common denominator $\left(1+x+\cdots+x^{m-1}\right)^{n}$ and hence, $r(x) \in R_{m n}$. Also, by (1),

$$
\begin{aligned}
\left|\boldsymbol{r}(x)-p_{n}(x)\right| & \leqq \sum_{k+i \leqq n} a_{k i} x^{k} i x^{m} \\
& \leqq n x^{m} \sum_{k+i \leqq n} a_{k i} x^{k} .
\end{aligned}
$$




$$
\begin{aligned}
\sum_{k+i \lesssim n} a_{k i} x^{k} & =\sum_{k+i \leqq n} a_{k i} x^{k} \frac{(1-x)^{i}}{(1-x)^{i}} \\
& \leqq \frac{1}{(1-x)^{n}} \sum_{k+i \leqq n} a_{k i} x^{k}(1-x)^{i}=\frac{p(x)}{(1-x)^{n}}
\end{aligned}
$$

we have

$$
\left|r(x)-p_{n}(x)\right| \leqq \frac{n x^{m} p(x)}{(1-x)^{n}}
$$

LEMMA 2. Suppose $p$ and $q$ are both P.P.C. of degree $n$. Then there exists $r \in R_{2 n m}^{++}$so that for any $x \in[0,1]$, satisfying $(1-x)^{n}>n x^{m}$,

$$
|p(x) / q(x)-r(x)| \leqq \frac{2 n x^{m}}{(1-x)^{n}-n x^{m}} \cdot \frac{p(x)}{q(x)} .
$$

Proof. By Lemma 1 we can choose $s$ and $t \in R_{n m}^{+++}$so that for $x \in[0,1)$,

$$
|p(x)-s(x)| \leqq \frac{n x^{m} p(x)}{(1-x)^{n}}
$$

and

$$
|q(x)-t(x)| \leqq \frac{n x^{m} q(x)}{(1-x)^{n}}
$$

Then, for $x \in[0,1)$,

$$
\begin{aligned}
\left|\frac{p(x)}{q(x)}-\frac{s(x)}{t(x)}\right| & =\left|\frac{p(x)}{q(x)}-\frac{s(x)}{q(x)}+\frac{s(x)}{q(x)}-\frac{s(x)}{t(x)}\right| \\
& \leqq\left|\frac{p(x)-s(x)}{q(x)}\right|+\left|\frac{s(x)(q(x)-t(x))}{t(x) q(x)}\right| \\
& \leqq \frac{n x^{m}}{(1-x)^{n}}\left|\frac{p(x)}{q(x)}\right|+\frac{n x^{m}}{(1-x)^{n}}\left|\frac{s(x)}{t(x)}\right| \\
& \leqq \frac{2 n x^{m}}{(1-x)^{n}}\left|\frac{p(x)}{q(x)}\right|+\frac{n x^{m}}{(1-x)^{n}}\left|\frac{p(x)}{q(x)}-\frac{s(x)}{t(x)}\right| .
\end{aligned}
$$

The result follows with $r=s / t$.

We now prove an analogue of Theorem 4 for rationals in the class R.P.C. Define a diamond-shaped region in the complex plane $G(\alpha)$ by

$$
G(\alpha)=\{z:|\arg (z)| \leqq \alpha\} \cap\{z:|\arg (1-z)| \leqq \alpha\} .
$$

LeMMA 3. Let $\varepsilon>0$. Suppose $p_{n} \in \Pi_{n}$ has no roots in the region $G(1 / h)$ and $p_{n}(x)>0$ for $x \in[0,1]$. Then $p_{n}(x)=r_{m}(x)$ where 
$r_{m}(x)$ is a R.P.C. of degree $m, m \leqq B_{\varepsilon} h^{(1+\varepsilon)} \cdot n$ and $B_{\varepsilon}$ is the same constant as appears in Theorem 4.

Proof. We write $p_{n}(x)=s_{k}(x) t_{n-k}(x)$ where $s_{k} \in \prod_{k}$ has no roots in $\{z:|\arg (z)| \leqq 1 / h\}$ and $t_{n-k} \in \Pi_{n-k}$ has no roots in $\{z:|\arg (1-z)| \leqq$ $1 / h\}$. By Theorem 4,

$$
s_{k}(x)=U_{j}(x) \in R_{j}^{++} \text {where } j \leqq B_{\varepsilon} h^{(1+\varepsilon)} k
$$

and since $t_{m-k}(x)=q_{m-k}(1-x)$ where $q_{m-k}(1-x)$ has no roots in $\{z: \arg (z) \leqq 1 / h\}$,

$$
t_{n-k}(x)=V_{i}(1-x) \text { where } V_{i}(x) \in R_{i}^{++} \text {and } i \leqq B_{\varepsilon} h^{(1+\varepsilon)}(n-k) .
$$

We set $r_{m}(x)=U_{j}(x) V_{i}(1-x)$ to complete the result.

LEMMA 4. Let $\varepsilon>0$. If $p_{n} \in \Pi_{n}$ has no roots in the region $G(1 / h)$ and $p_{n}(x)>0$ for $x \in[0,1]$, then there exists $r \in R_{2 c m n}^{++}$where $c=B_{\varepsilon} h^{(1+\varepsilon)}$ so that for $x \in[0,1)$,

$$
|p(x)-r(x)| \leqq \frac{2 n x^{m}|p(x)|}{(1-x)^{c n}-c n x^{m}}
$$

provided $(1-x)^{c n} \geqq c n x^{m}$.

Proof. By Lemma 3, there exists $s$ an R.P.C. of degree at most $c n=B_{\varepsilon} h^{(1+s)} n$ so that $p=s$. By Lemma 2, there exists $r \in$ $R_{2 c n m}^{++}$so that

$$
|p(x)-r(x)|=|s(x)-r(x)| \leqq \frac{2 n x^{m}|p(x)|}{(1-x)^{c n}-c n x^{m}} .
$$

4. Approximating analytic functions. Let $\rho>1$ and let $E_{\rho}$ be the closed ellipse in the complex plane with foci at 0 and 1 and with semiaxes $1 / 4\left(\rho+\rho^{-1}\right)$ and $1 / 4\left|\rho-\rho^{-1}\right|$. S.N. Bernstein proved:

THEOREM 5. ([2] p. 76.) If $f$ is analytic on $E_{\rho}$ then there exist polynomials $p_{n} \in \Pi_{n}$ so that

$$
\left\|f-p_{n}\right\|_{[0,1]}=0\left(1 / \rho^{n}\right)
$$

and $p_{n} \rightarrow f$ uniformly on $E_{\rho}$.

We show that positive analytic functions can be approximated almost as efficiently by rational functions from the class R.P.C.

THEOREM 6. If $f$ is analytic and never zero on $E_{\rho}$ and $f(x)>0$ for $x \in[0,1]$, then there exists a sequence of $r_{n} \in$ R.P.C., $r_{n}$ of degree 
$n$, so that for each $\varepsilon>0$,

$$
\left\|f-r_{n}\right\|_{[0,1]}=O\left(1 / \rho^{n / c_{\varepsilon}}\right)
$$

where $c_{\varepsilon}=B_{\varepsilon}\left[\left(\tan ^{-1}\left(\rho+\rho^{-1}\right) / 2\right)\right]^{-(1+\varepsilon)}$ and $B_{\varepsilon}$ is the same constant as in Theorem 4.

Proof. By Theorem 5 there exists a sequence of polynomials $p_{n}$ so that

$$
\left\|f-p_{n}\right\|_{[0,1]}=O\left(1 / \rho^{n}\right)
$$

and each $p_{n}$ has no zeros on $E_{\rho}$. We note that the region

$$
G\left(\tan ^{-1}\left(\frac{\rho+\rho^{-1}}{2}\right)\right) \subset E_{\rho}
$$

and hence, by Lemma 3,

$$
p_{n}=r_{m} \in \text { R.P.C. where } m \leqq B_{\varepsilon}\left(\tan ^{-1}\left(\frac{\rho+\rho^{-1}}{2}\right)\right)^{-(1+\varepsilon)} \cdot n \text {. }
$$

The result is finished by substituting $r_{m}$ into (1).

We have the following two theorems for approximating analytic functions by rational functions with positive coefficients.

THEOREM 7. Let $0<\rho<1$. If $f$ is analytic and never zero on $E_{\rho}$ and $f(x)>0$ for $x \in[0,1]$, then there exists a constant $\gamma$ so that

$$
R_{n}^{++}(f:[0, \rho])=O\left(1 / \gamma^{\sqrt{n}}\right)
$$

where $\gamma$ depends only on $\rho$ and $\delta$.

Under stronger assumptions on $f$ we recover exponential rates of convergence.

TheOREM 8. Let $0<\delta<1$. Suppose that $f(z)=\Sigma a_{k} z^{k}, a_{k}$ real, is analytic in a region containing $\{z:|z| \leqq 1\}$ and suppose that

$$
f(x)>0 \text { for } x \in[0,1] \text {. }
$$

Then there exists $\eta>1$ so that

$$
B_{n}^{++}(f:[0, \delta])=0\left(1 / \eta^{n}\right)
$$

where $\eta$ is independent of $n$.

Proof of Theorem 7. By Theorem 4, there exists a sequence of polynomials $p_{n} \in \Pi_{n}$ so that 


$$
\left\|f-p_{n}\right\|_{[0,1]}=O\left(1 / \rho^{n}\right)
$$

where each $p_{n}$ has no roots in $E_{\rho}$. Since $G\left(\tan ^{-1}\left[\left(\rho+\rho^{-1}\right) / 2\right] \subset E_{\rho}\right.$ we deduce, from Lemma 4 with $h=1 / \tan ^{-1}\left[\left(\rho+\rho^{-1}\right) / 2\right]$ and $m=i n$, that there exists $r_{k_{n}} \in R_{2 i c n^{2}}^{++}$so that

$$
\left|p_{n}-r_{k_{n}}\right| \leqq \frac{2 n x^{i n}\|p\|_{[0,1]}}{(1-x)^{c n}-c n x^{i n}} .
$$

From (1) and (2) we have, for fixed $i$ sufficiently large,

$$
\left\|f-r_{k_{n}}\right\|_{[0, \hat{o}]}=0\left[\frac{1}{\rho^{n}}+\frac{n \delta^{i n}}{(1-\delta)^{c_{n}}}\right] .
$$

Since $k_{n} \leqq 2 i c n^{2}$, the result follows.

We need the next lemma in the proof of Theorem 8. Let $D_{\alpha}$ be the open disc of radius $\alpha$ centered at the origin.

LEMMA 5. Let $\beta>\alpha$. Suppose $f(z)=\sum_{k=0}^{\infty} a_{k} z^{k}$ is analytic on $D_{\beta}$. Then, for $z \in D_{\alpha}$,

$$
f(z)=\frac{\sum_{k=0}^{\infty}\left(s_{k}(f: \alpha) / \alpha^{k}\right) z^{k}}{\sum_{k=0}^{\infty} z^{k} / \alpha^{k}}
$$

where $s_{k}(f: \alpha)$ is the kth Taylor polynomial of $f$ evaluated at $\alpha$.

Proof. Let

$$
g(z)=\frac{1}{1-z / \alpha}=\sum_{k=0}^{\infty} z^{k} / \alpha^{k}
$$

Then,

$$
\begin{aligned}
f(z) g(z) & =\sum_{k=0}^{\infty}\left(\sum_{m=0}^{k} \frac{a_{m}}{\alpha^{k-m}}\right) z^{k} \\
& =\sum_{k=0}^{\infty} \frac{1}{\alpha^{k}}\left(\sum_{m=0}^{k} a_{m} \alpha^{m}\right) z^{k} \\
& =\sum_{k=0}^{\infty}\left[\frac{s_{k}(f: \alpha)}{\alpha^{k}}\right] z^{k}
\end{aligned}
$$

Proof of Theorem 8. By assumption, $f$ is analytic in some disc $D_{\beta}$ where $\beta>1$. Setting $\alpha=1$ in Lemma 5 yields, for $z \in D_{1}$,

$$
f(z)=\frac{\sum_{k=0}^{\infty} s_{k}(f: 1) z^{k}}{\sum_{k=0}^{\infty} z^{k}} .
$$


Since $f(x)>0$ for $x \in[0,1]$, there exist $N$ so that for $n \geqq N$, $s_{n}(f: 1)>0$ and so that $\sum_{k=0}^{N} s_{k}(f: 1) x^{k}$ is strictly positive on $[0, \infty]$. For $m \geqq N$ set

$$
r_{m}(z)=\frac{\sum_{k=0}^{N} s_{k}(f: 1) z^{k}}{\sum_{k=0}^{m} z^{k}}+\frac{\sum_{k=N+1}^{m} s_{k}(f: 1) z^{k}}{\sum_{k=0}^{m} z^{k}} .
$$

The second term of the right side of (1) is an element of $R_{m}^{++}$. The first term has a fixed numerator which is positive on $[0, \infty]$ and by Theorem 4, there exists a constant $A$, independent of $m$, so that this term is an element of $R_{A m}^{++}$. Thus, there exists $A$ so that for each $m \geqq N$

$$
r_{m} \in R_{A m}^{++} .
$$

We finish the proof by observing that

$$
\begin{aligned}
\left\|f-r_{m}\right\|_{[0, \hat{o}]} & =\left\|\frac{\sum_{k=0}^{\infty} s_{k}(f: 1) z^{k}}{\sum_{k=0}^{\infty} z^{k}}-\frac{\sum_{k=0}^{m} s_{k}(f: 1) z^{k}}{\sum_{k=0}^{m} z^{k}}\right\|_{[0, \delta]} \\
& \leqq\left|\sum_{k=m+1}^{\infty} s_{k}(f: 1) \delta^{k}\right|+\|f\|_{[0, \delta]} \cdot\left|\sum_{k=m+1}^{\infty} \delta^{k}\right| \\
& =O\left(\delta^{m}\right) .
\end{aligned}
$$

5. Approximating continuous functions. We prove the following three theorems:

THEOREM 9. If $f \in C[0,1 / 2]$ and $f \geqq 0$ on $[0,1 / 2]$ then

$$
R_{m n}^{++}(f:[0,1 / 2]) \leqq\|f\|_{[0,1 / 2]} n 2^{n-m}+2 \omega(f, 1 / \sqrt{n}) .
$$

THEOREM 10. If $f \in C[0,1 / 2], f \geqq 0$ on $[0,1 / 2]$ then for each $\delta>0$ there exists $A_{\delta}$ depending only on $\delta$ so that

$$
R_{n}^{++}(f:[0,1 / 2]) \leqq A_{\dot{\delta}} \omega\left(f, 1 / n^{1 /(4+\delta)}\right) .
$$

Theorem 11. If $f \in C^{k}[0,1 / 2], f>0$ on $[0,1 / 2]$ and $f^{(k)} \in \operatorname{lip}^{\top} \alpha$, $0<\alpha<1$, then for each $\delta>0$ there exists $A_{\delta}$ so that

$$
R_{n}^{++}(f:[0,1 / 2]) \leqq A_{\delta}\left[\frac{1}{n^{1 /(4+\delta)}}\right]^{k+\alpha},
$$

where $A_{\delta}$ is independent of $n$.

We have use the notation $\omega(f, \cdot)$ for the modulus of continuity 
of $f$.

We now collect the results we need to prove the above theorems. For $f \in C[0,1]$ we define the $n$th Bernstein polynomial by

$$
B_{n}(x)=B_{n}(f: x)=\sum_{k=0}^{n} f(k / n)\left(\begin{array}{l}
n \\
k
\end{array}\right) x^{k}(1-x)^{n-k} .
$$

THEOREM 12. ([5] p. 15.) If $f \in C[0,1]$ then

$$
\left\|f(x)-B_{n}(f: x)\right\|_{[0,1]} \leqq 2 \omega(f, 1 / \sqrt{n}) .
$$

Theorem 13 (Lorentz [1].) If $f \in C^{k}[0,1], f>0$ on $[0,1]$ and $f^{(k)} \in \operatorname{lip} \alpha, 0<\alpha \leqq 1$, then there exists $p_{n}$ a P.P.C. of degree $n$ so that

$$
\left\|f(x)-p_{n}(x)\right\|_{[0,1]} \leqq C\left(\frac{1}{\sqrt{n}}\right)^{k+\alpha}
$$

where $C$ is independent of $n$.

Proof of Theorem 9. We extend $f$ to a continuous function on $[0,1]$ by setting, for $x \in[0,1 / 2]$

$$
f\left(x+\frac{1}{2}\right)=f\left(x-\frac{1}{2}\right) .
$$

Then the modulus of continuity of $f$ on $[0,1]$ is the same as the modulus of continuity of $f$ on $[0,1 / 2]$.

Consider $B_{n}$ the $n$th Bernstein polynomial for $f$. Since $f$ is nonnegative on $[0,1], B_{n}$ is a P.P.C. of degree $n$ and $\left\|B_{n}\right\|_{[0,1 / 2]} \leqq$ $\|f\|_{[0,1]}$. Thus, by Lemma 1 with $x \leqq 1 / 2$ and Theorem 12,

$$
\begin{aligned}
R_{m n}^{++}\left(f:\left[0, \frac{1}{2}\right]\right) & \leqq R_{m n}^{++}\left(B_{n}:\left[0, \frac{1}{2}\right]\right)+\left\|B_{n}-f\right\|_{[0,1 ; 2]} \\
& \leqq\|f\|_{[0,1]} n 2^{n-m}+2 \omega(f, 1 / \sqrt{n}) .
\end{aligned}
$$

Theorem 10 is a corollary to Theorem 9. We observe that it suffices to prove Theorem 10 under the assumption that $f$ has a zero on $[0,1 / 2]$ and that under this assumption $2 \omega(f, 1 / \sqrt{n}) \geqq(1 / n)\|f\|_{[0,1]}$. The result is now completed by choosing $m=n^{\delta}$ for small $\delta$, and applying Theorem 9 .

Theorem 11 is proved analogously to Theorems 9 and 10 . We first extend $f$ to $[0,1]$ in such a way that $f>0$ on $[0,1]$ and so that $f \in C^{k}[0,1]$ with $f^{(k)} \in \operatorname{lip} \alpha$. We now approximate this extended $f$ by a P.P.C. as guaranteed by Theorem 13 and proceed as in the proofs of Theorem 9 and Theorem 10. 
6. Remarks.

(1) D. J. Newman and A. R. Reddy [4] show that the best approximant to $x^{n+1}$ from $R_{n}^{++}$on $[0,1]$ is a monomial $\alpha x^{n}$ and that

$$
R_{n}^{++}\left(x^{n+1}:[0,1]\right)=\prod_{n}^{+}\left(x^{n+1}:[0,1]\right) \sim c / n \text {. }
$$

This should be compared to the fact ([2] p. 31) that

$$
\Pi_{n}\left(x^{n+1}:[0,1]\right)=\frac{1}{2^{2 n+1}}
$$

(2) The restriction that $f$ be strictly positive is essential in Theorems 7 and 11.

LEMMA 6. Let $0<\alpha<\beta$. If $f(\alpha)=0$

$$
R_{n}^{++}(f:[\alpha, \beta]) \geqq \frac{f(\beta)}{\left(1+\beta^{n} / \alpha^{n}\right)} .
$$

Proof. Let $p_{n} / q_{n}$ be a best approximant to $f$ from $R_{n}^{++}$on $[\alpha, \beta]$. Then we can write

$$
p_{n}(x)=\sum_{k=0}^{n} a_{k} x^{k} \text { where } a_{k} \geqq 0
$$

We have

$$
p_{n}(\beta)=\sum_{k=0}^{n} a_{k} \beta^{k}=\sum_{k=0}^{n} \frac{\beta^{k}}{\alpha^{k}} a_{k} \alpha^{k} \leqq \frac{\beta^{n}}{\alpha^{n}} p_{n}(\alpha)
$$

and hence,

$$
\begin{aligned}
R_{n}^{++}(f:[\alpha, \beta]) & \geqq f(\beta)-\frac{p_{n}(\beta)}{q_{n}(\beta)} \\
& \geqq f(\beta)-\frac{\beta^{n}}{\alpha^{n}} \frac{p_{n}(\alpha)}{q_{n}(\alpha)}
\end{aligned}
$$

Since

$$
\frac{p_{n}(\alpha)}{q_{n}(\alpha)} \leqq f(\alpha)+R_{n}^{++}(f:[\alpha, \beta])
$$

we have

$$
R_{n}^{++}(f:[\alpha, \beta]) \geqq f(\beta)-\frac{\beta^{n}}{\alpha^{n}} R_{n}^{++}(f:[\alpha, \beta])
$$

Suppose that $f$ is continuous on $[0,1]$ and $f(1 / 2)=0$. If we set $\alpha=1 / 2$ and $\beta=1 / 2+1 / 2 n$ in Lemma 6 then 


$$
R_{n}^{++}(f:[0,1]) \geqq R_{n}^{++}\left(f:\left[\frac{1}{2}, \frac{1}{2}+\frac{1}{2} n\right]\right) \geqq \frac{f(1 / 2+1 / 2 n)}{(1+e)}
$$

In particular

$$
R_{n}^{+}+\left(\left(x-\frac{1}{2}\right)^{2}:[0,1]\right) \geqq \frac{1}{4 n^{2}} \frac{1}{(1+e)}
$$

7. Acknowledgment. Some of the results of the paper are included in the author's doctoral dissertation at the University of British Columbia under the direction of Dr. David Boyd, to whom the author wishes to express his gratitude.

\section{REFERENCES}

1. G. G. Lorentz, The degree of approximation by polynomials with positive coefficients, Math. Ann., 151 (1963), 239-251.

2. - Approximation of Functions, New York: Holt, Rinehart and Winston, 1966.

3. E. Meissner, Über positive Darstellungen von Polynomen, Math. Ann., 70 (1911), 223-255.

4. D. J. Newman and A. R. Reddy, Rational approximation to $x^{n}$, Pacific J. Math., 67 (1976), 247-250.

5. T. J. Rivlin, An Introduction to the Approximation of Functions, Waltham, Mass., Toronto, London: Blaisdell Publishing Co., 1969.

Received July 19, 1978.

The University of British Columbia

Vancouver, B. C. Canada V6T 1W5 


\section{PACIFIC JOURNAL OF MATHEMATICS}

\section{EDITORS}

DONALD BABBITT (Managing Editor)

University of Galifornia

Los Angeles, California 90024

Hugo Rossi

University of Utah

Salt Lake City, UT 84112

C. C. MOORE AND ANDREW OGG

University of California

Berkeley, CA 94720
J. DugundjI

Department of Mathematics University of Southern California Los Angeles, California 90007

R. Finn and J. Milgram Stanford University Stanford, California 94305

ASSOCIATE EDITORS

E. F. BECKENBACH

B. H. NeumanN

F. WOLF

K. YoSHIDA

\section{SUPPORTING INSTITUTIONS}

UNIVERSITY OF BRITISH COLUMBIA

UNIVERSITY OF SOUTHERN CALIFONIA

CALIFORNIA INSTITUTE OF TECHNOLOGY

UNIVERSITY OF CALIFORNIA

MONTANA STATE UNIVERSITY

STANFORD UNIVERSITY

UNIVERSITY OF HAWAII

UNIVERSITY OF NEVADA, RENO

UNIVERSITY OF TOKYO

NEW MEXICO STATE UNIVERSITY

UNIVERSITY OF UTAH

OREGON STATE UNIVERSITY

WASHINGTON STATE UNIVERSITY

UNIVERSITY OF OREGON

UNIVERSITY OF WASHINGTON 


\section{Pacific Journal of Mathematics \\ Vol. 88, No. $1 \quad$ March, 1980}

Michael James Beeson, Extensionality and choice in constructive mathematics .................................... 1

José L. Blasco Olcina, Two questions on Wallman rings.............. 29

Peter I. Booth and J. Tillotson, Monoidal closed, Cartesian closed and convenient categories of topological spaces .................. 35

Peter B. Borwein, Rational functions with positive coefficients, polynomials and uniform approximations ......................... 55

Josip Globevnik, Fourier coefficients of the Rudin-Carleson extensions . . . . 69

Jacob Kofner, Quasimetrizable spaces ...................... 81

Mark Mandelker, Resolutions on the line ..................... 91

Lynn McLinden, An analogue of Moreau's proximation theorem, with application to the nonlinear complementarity problem ............ 101

Atsushi Murase, On the uniform distribution property of certain linear algebraic groups ................................ 163

Nicholas Th. Varopoulos, Zeros of $H^{p}$ functions in several complex

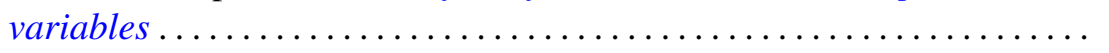

